United Nations Educational Scientific and Cultural Organization and

International Atomic Energy Agency

THE ABDUS SALAM INTERNATIONAL CENTRE FOR THEORETICAL PHYSICS

\title{
THE ALBANESE MAP OF A 3-FOLD OF GENERAL TYPE WHOSE CANONICAL MAP IS COMPOSED WITH A PENCIL
}

\author{
Jin-Xing Cai ${ }^{1}$ \\ School of Mathematical Sciences, Peking University, \\ Beijing 100871, People's Republic of China \\ and \\ The Abdus Salam International Centre for Theoretical Physics, Trieste, Italy.
}

\section{MIRAMARE - TRIESTE}

July 2000

\footnotetext{
${ }^{1}$ E-mail: cai@ictp.trieste.it; caijx@sxx0.math.pku.edu.cn
} 


\section{INTRODUCTION}

Let $X$ be a complex projective manifold of general type. A natural question is, if $X$ enjoys such and such analytic properties, then how is the topology of $X$ ? In this note we will study some topological properities of $X$ when the canonical map $\Phi_{X}$ of $X$ is composed with a pencil.

It is known that, if $X$ is a surface of general type whose canonical map is composed with a pencil, then the irregularity $q(X) \leq 2[\mathrm{X} 1]$. (We refer the reader to [Be1] in which the canonical map of surface of general type was first systematically studied.) However, when $\operatorname{dim} X \geq 3$, simple examples show that $q(X)$ can be arbitrarily large whenever $\Phi_{X}$ is composed with a pencil.

We recall that, the Albanese dimension $a(X)$ of a manifold $X$, is defined to be the dimension of the image of the Albanese map alb: $X \rightarrow \operatorname{Alb}(X)$, and $X$ is said of maximal Albanese dimension or of Albanese general type if $a(X)=\operatorname{dim} X$. We note that $a(X)$ is a topological invariant of $X$ (cf. [Ca, Proposition 1.4]). Our main result is the following.

Theorem 1. Let $X$ be a smooth projective 3-fold of general type over the complex number field. Assume that the canonical linear system is composed with a pencil, and the irregularity $q(X) \geq 6$. Then $X$ is not of maximal Albanese dimension.

The condition that $q(X) \geq 6$ is technical (see Remark below). Examples of 3-folds $X$ with i) $\operatorname{dim}\left(\operatorname{Im} \Phi_{X}\right)=2$ and $a(X)=3$, and ii) $\operatorname{dim}\left(\operatorname{Im} \Phi_{X}\right)=1$ and $a(X)=2$ are easily constructed (see Example 1). Theorem 1 will be proved in section 1 . In section 2 we will consider the behaviour of the canonical map under smooth deformations.

Theorem 2. Let $X$ be a smooth projective 3-fold of general type whose canonical map is composed with a pencil. Let $f: X \rightarrow C$ be the fiber space associated to the canonical map. If $g(C) \geq 2$, then the canonical map of any (global) smooth deformation of $X$ is composed with a pencil.

We use standard notations as in [BPV] or [Mo].

Acknowledgments. I am grateful to the referee for helpful suggestions. This work is supported by the Abdus Salam International Centre for Theoretical Physics, Trieste, Italy and partially supported by the National Natural Science Foundation of China.

\section{Proof of Theorem 1}

Let $X$ be a smooth complex projective 3 -fold with $p_{g}(X) \geq 2$. The canonical map

$$
\Phi_{X}: X--\rightarrow \mathbb{P}^{p_{g}(X)-1}
$$

is defined by the moving part of the canonical linear system $\left|K_{X}\right|$. Assume that $\Phi_{X}$ is composed with a pencil. Let $\pi: X^{\prime} \rightarrow X$ be a resolution of the base locus of the moving part of $\left|K_{X}\right|$. 
We have the following commutative diagram

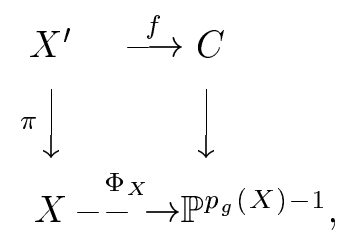

where $f: X^{\prime} \rightarrow C$ is the Stein factorization of $\Phi_{X} \circ \pi$. We call $f: X^{\prime} \rightarrow C$ the canonical fibration associated with the canonical map. Clearly, such a fibration is defined up to birational equivalence. But this does not matter, since we only consider birational invariants (e.g., the Albanese map, a $(X), q(X)$, etc.). Using $X^{\prime}$ instead of $X$, for convenience, such a canonical fibration is denoted by $f: X \rightarrow C$.

Proposition 1. (cf. $[\mathrm{CC}]$ ) Let $X$ be a 3-fold with $p_{g}(X) \geq 2$. Assume that the canonical map $\Phi_{X}$ is composed with a pencil. Let $f: X \rightarrow C$ be the fiber space associated with $\Phi_{X}$, and $F$ a general fiber of $f$. If $g(C) \geq 2$, then we have that either $p_{g}(F)=1$ or $p_{g}(F)=p_{g}(X)=$ $g(C)=2$.

Proof. Since $p_{g}(X) \neq 0$, we have $p_{g}(F)>0$. Now we assume that $p_{g}(F) \geq 2$. Let $M$ and $E$ be the moving part and the fixed part of $\left|K_{X}\right|$ respectively. Since $\Phi_{X}$ factors through $f$, $\mathcal{O}_{X}(M)=f^{*} \mathcal{L}^{\prime}$ for some $\mathcal{L}^{\prime} \in \operatorname{Pic} C$. Thus $h^{0}\left(\mathcal{L}^{\prime}\right)=p_{g}(X)$ and $\omega_{X}=f^{*} \mathcal{L}^{\prime} \otimes \mathcal{O}_{X}(E)$. There is an injective map of sheaves $\mathcal{L}^{\prime} \hookrightarrow f_{*} \omega_{X}$. Let $\mathcal{L}$ be the saturated subsheaf of $\mathcal{L}^{\prime}$ in $f_{*} \omega_{X}$. Then $\mathcal{L}$ is invertible and the quotient $\mathcal{Q}:=f_{*} \omega_{X} / \mathcal{L}$ is torsion free, hence locally free of rank $p_{g}(F)-1$. We have an exact sequence of sheaves

$$
0 \rightarrow \mathcal{L} \rightarrow f_{*} \omega_{X} \rightarrow \mathcal{Q} \rightarrow 0
$$

Consider the natural map $H^{0}\left(\omega_{C} \otimes \mathcal{L}^{-1}\right) \times H^{0}(\mathcal{L}) \rightarrow H^{0}\left(\omega_{C}\right)$, note that $h^{0}(\mathcal{L}) \geq h^{0}\left(\mathcal{L}^{\prime}\right) \geq 2$, by [Ha, Lemma IV.5.5]. We have

$$
\begin{aligned}
g(C)-1 & \geq h^{0}\left(\omega_{C} \otimes \mathcal{L}^{-1}\right) \\
=h^{1}(\mathcal{L}) & \geq h^{0}(\mathcal{Q}) \quad\left(\text { note that } h^{0}\left(f_{*} \omega_{X}\right)=h^{0}(\mathcal{L})\right) \\
& \geq\left(p_{g}(F)-1\right)(g(C)-1) .
\end{aligned}
$$

The last inequality follows by the Riemann-Roch Theorem and the semipositivity of $f_{*} \omega_{X / C}=$ $f_{*} \omega_{X} \otimes \omega_{C}^{-1}$ (cf. [Fu]). This implies $p_{g}(F)=2$ and $h^{1}(\mathcal{L})=g(C)-1$. Now by Clifford's theorem, we have $\operatorname{deg} \mathcal{L}=2$ and $\mathcal{L} \simeq \omega_{C}$. So $p_{g}(X)=g(C)=2$.

Lemma 1. Let $X$ be a 3-fold of general type whose canonical map is composed with a pencil, and $f: X \rightarrow C$ the fiber space associated with $\Phi_{X}$. If $q(X) \geq 5$ and $g(C) \geq 2$, then alb: $X \rightarrow$ $\operatorname{Alb}(X)$ is not of Albanese general type.

Proof. Let $F$ be a general fiber of $f$. We have $q(X)-g(C) \leq q(F)$. Since $F$ is (a surface) of general type, $q(F) \leq p_{g}(F)$. If $g(C) \geq 2$, by Proposition 1 , we have that either $q(X)-g(C) \leq 1$ 
or $q(X) \leq g(C)+q(F) \leq 2+p_{g}(F)=4$. Thus $f^{*} H^{0}\left(\Omega_{C}^{1}\right)$ is of codimension $\leq 1$ in $H^{0}\left(\Omega_{X}^{1}\right)$ by the assumption. So for any $\alpha, \beta, \gamma \in H^{0}\left(\Omega_{X}^{1}\right)$, we have $\alpha \wedge \beta \wedge \gamma=0$. Note that $X$ is of Albanese general type if and only if Image $\left(\stackrel{3}{\wedge} H^{0}\left(\Omega_{X}^{1}\right) \rightarrow H^{0}\left(\omega_{X}\right)\right) \neq 0$. (cf. [Ca])

Notation. Let $X$ be a complex projective manifold. For any $0 \neq \alpha \in H^{0}\left(\Omega_{X}^{n}\right)(1 \leq n \leq$ $\operatorname{dim} X)$, we denote by $\mathrm{Z}(\alpha)$ the zero-locus of the holomorphic $n$-form $\alpha$. When $n=\operatorname{dim} X$, we have $\mathrm{Z}(\alpha)=\operatorname{div}(\alpha)$.

Lemma 2. Let $f: X \rightarrow C$ be as in Lemma 1. Assume that $g(C) \leq 1$, and that there exist linearly independent 1 -forms $\alpha, \beta \in H^{0}\left(\Omega_{X}^{1}\right)$, such that $\alpha \wedge \beta=0$. Then alb: $X \rightarrow \operatorname{Alb}(X)$ is not of Albanese general type.

Proof. By a well-known theorem of Castelnuovo-de Franchis (see e.g. [B-P-V]), there exists a surjective morphism $h: X \rightarrow B$ to a curve $B$ (of genus $\geq 2$ ) such that $\alpha, \beta \in h^{*} H^{0}\left(\Omega_{B}^{1}\right)$. Let $H$ be a general fiber of $h$. Then $H$ is not contained in the fixed part of $\left|K_{X}\right|$. Since $g(C) \leq 1$ by the assumption, $f$ and $h$ are different fibrations of $X$. So $H$ is not contained in the moving part of $\left|K_{X}\right|$. Since $\alpha, \beta \in h^{*} H^{0}\left(\Omega_{B}^{1}\right)$, we have that $H \subset Z\left(t \alpha+t^{\prime} \beta\right)\left(t \alpha+t^{\prime} \beta \neq 0\right)$ for a suitable choice $t, t^{\prime} \in \mathbb{C}$. If a $(X)=3$, then there exist two 1 -forms $\gamma_{1}, \gamma_{2} \in H^{0}\left(\Omega_{X}^{1}\right)$ such that $\omega:=\left(t \alpha+t^{\prime} \beta\right) \wedge \gamma_{1} \wedge \gamma_{2} \neq 0$. Then $H \subset \operatorname{div}(\omega) \in\left|K_{X}\right|$. This is a contradiction.

\section{Proof of Theorem 1.}

Notations as in Lemma 1. By Lemma 1, we can assume that $g(C) \leq 1$. We consider the Albanese map alb: $X \rightarrow \operatorname{alb}(X) \subset \operatorname{Alb}(X)$. Suppose that $X$ is of Albanese general type.

If $\kappa(\operatorname{alb}(X))=3$, then $\Phi_{\mathrm{alb}(X)}$ and hence $\Phi_{X}$ is generically finite by a theorem of Griffiths and Harris (cf. [Mo, Theorem 3.9]). This contradicts the assumption that $\Phi_{X}$ is composed with a pencil.

Since $\operatorname{alb}(X)$ is a proper subvariety of $\operatorname{Alb}(X)$ generating $\operatorname{Alb}(X)$, one has that $\kappa(\operatorname{alb}(X))>$ 0 . By a theorem of Ueno [Ue, Theorem 10.9], there exist an abelian subvariety $A$ of $\operatorname{Alb}(X)$ and a projective variety $S^{\prime}$ which is a subvariety of an abelian variety $\operatorname{such}$ that $\operatorname{alb}(X)$ is a fiber bundle over $S^{\prime}$ (denoted by $u^{\prime}$ ) whose fiber is $A$, and $\kappa\left(S^{\prime}\right)=\operatorname{dim}\left(S^{\prime}\right)=\kappa(\operatorname{alb}(X))$.

Let

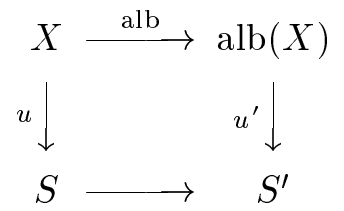

be the Stein factorization of $u^{\prime} \circ$ alb. Since the invariants we consider are birational, by a standard argument, we can assume that $S$ is smooth.

If $\kappa(\operatorname{alb}(X))=1$, then $S$ is a curve of genus $\geq 2$. This is impossible by Lemma 2 .

In what follows, we treat the remaining case when $\kappa(\operatorname{alb}(X))=2$. In this case, $S$ is a surface of general type, and $q(S)=q(X)-1$. (Note that $q(\operatorname{alb}(X))-q\left(S^{\prime}\right) \leq q(A)=1$, and if $q(S)=q(X)$ then $X$ is not of Albanese general type.) 
We distinguish two cases according to whether $f$ factors through $u$ or not.

Case 1. $f$ does not factor through $u$.

Let $s \in S$ be a general point such that

i) $u^{-1} s$ is not contained in the fixed part of $\left|K_{X}\right|$, and

ii) $\left.f\right|_{u^{-1} s}: u^{-1} s \rightarrow C$ is surjective.

Since $q(S)>3$, there exists $0 \neq \alpha \in H^{0}\left(\Omega_{S}^{1}\right)$ such that $s \in Z(\alpha)$. We have that $u^{-1} s \subset$ $Z\left(u^{*} \alpha\right)$. Let $0 \neq \beta \in H^{0}\left(\Omega_{X}^{1}\right) \backslash u^{*} H^{0}\left(\Omega_{S}^{1}\right)$, and $\gamma \in H^{0}\left(\Omega_{S}^{1}\right)$ such that $u^{*} \alpha, \beta, u^{*} \gamma$ are linearly independent. By Lemma 2, we can assume that $\alpha \wedge \gamma \neq 0$. Since $\beta$ is closed, it is easy to verify that $\omega:=\beta \wedge u^{*} \alpha \wedge u^{*} \gamma \neq 0$. Now $u^{-1} s \subset \operatorname{div}(\omega) \in\left|K_{X}\right|$. This implies that there exists an irreducible component $\Gamma$ of $\operatorname{div}(\omega)$ such that $u^{-1} s \subset \Gamma$. Clearly $\Gamma$ is not a fiber of $f$ since $\left.f\right|_{u^{-1} s}: u^{-1} s \rightarrow C$ is surjective by the choice of $s$. So $\Gamma$ is an irreducible component of the fixed part of $\left|K_{X}\right|$. This is a contradiction.

Case 2. $f$ factors through $u$.

Let $h: S \rightarrow C$ be the rational map such that $f=h \circ u$. By a suitable choice of the birational model of $S$ and hence of $X$, we can assume that $h$ is a morphism.

We prove the case when $g(C)=1$. The proof of the case when $g(C)=0$ is similar and left to the reader.

Let $s_{1}, s_{2} \in S$ be general points, such that

i) $h\left(s_{1}\right) \neq h\left(s_{2}\right)$ and $F_{i}=f^{*}\left(h\left(s_{i}\right)\right)(i=1,2)$ are general fibers of $f$;

ii) $u^{-1} s_{i}(i=1$ and 2$)$ is not contained in the fixed part of $\left|K_{X}\right|$.

Since $q(S)=q(X)-1 \geq 5$, we have that there exists $0 \neq \alpha \in H^{0}\left(\Omega_{S}^{1}\right)$ such that $s_{i} \in Z(\alpha)$ for $i=1$ and 2. If $F_{i} \subset Z\left(u^{*} \alpha\right)$ for $i=1$ and 2, then $f^{*} t \cdot u^{*} \alpha$ is a holomorphic 1-form on $X$, where $t$ is a meromorphic function having simple poles exactly at $h\left(s_{1}\right)$ and $h\left(s_{2}\right)$. Clearly $f^{*} t \cdot u^{*} \alpha$ and $u^{*} \alpha$ are linearly independent and $\left(f^{*} t \cdot u^{*} \alpha\right) \wedge u^{*} \alpha=0$. By Lemma 2, we get a contradiction.

Now we can assume that $F_{i} \not \subset Z\left(u^{*} \alpha\right)$ for some $i$ (say $i=1$ ). Let $p \in F_{1}$ be a general point. Then $p \notin \mathrm{Z}\left(u^{*} \alpha\right)$. Let $0 \neq \beta \in H^{0}\left(\Omega_{X}^{1}\right) \backslash u^{*} H^{0}\left(\Omega_{S}^{1}\right)$, and $\gamma_{1}, \gamma_{2} \in H^{0}\left(\Omega_{S}^{1}\right)$ such that $\gamma_{1}, \gamma_{2}, \alpha$ are linearly independent. By Lemma 2 , we can assume that $\alpha \wedge\left(t_{1} \gamma_{1}+t_{2} \gamma_{2}\right) \neq 0$ for any linear combination $t_{1} \gamma_{1}+t_{2} \gamma_{2} \neq 0$. Then we have that $\omega_{i}:=\beta \wedge u^{*} \alpha \wedge u^{*} \gamma_{i} \neq 0$, and $\omega_{i}(i=1$ and 2$)$ are $\mathbb{C}$-linearly independent. Thus $\operatorname{div}\left(\omega_{i}\right)(i=1$ and 2$)$ generate a linear pencil $\Lambda$ of $X$. Since $p$ is a general point of $F_{1}$ and $F_{1}$ is a general fiber of $f$, we can assume that $p$ is not in the base locus of $\Lambda$. So we have that $p \notin \operatorname{div}\left(\omega_{i}\right)$ for some $i$. Fix such an $i$. Since $u^{-1} s_{1} \subset \operatorname{div}\left(\omega_{i}\right) \in\left|K_{X}\right|$, there exists an irreducible component $\Gamma$ of $\operatorname{div}\left(\omega_{i}\right)$ such that $u^{-1} s \subset \Gamma$. By the above argument, $\Gamma \neq F_{i}$. So $\Gamma$ is a component of the fixed part of $\left|K_{X}\right|$. This contradicts the choice of $s_{1}$.

Corollary. Let $X$ be as in Theorem 1. Assume that the canonical map $\Phi_{X}$ is composed with a pencil, and $q(X) \geq 6$. Let $f: X \rightarrow C$ be the fiber space associated with $\Phi_{X}$. Assume that $g(C) \geq 2$. Then $\kappa(\operatorname{alb}(X))=1$. 
Proof. By Theorem 1 we have $\mathrm{a}(X) \leq 2$. The corollary is trivial when $\mathrm{a}(X)=1$.

Now we assume that $\operatorname{alb}(X)$ is a surface. We claim that $f$ factors through alb. Otherwise, there is a dominant rational map $X--\rightarrow C \times \operatorname{alb}(X)$, which implies $q(X) \geq q(\operatorname{alb}(X))+g(C)$. We get necessarily $g(C)=0$, contradicting the assumption. Let $F$ be a general fiber of $f$. Since $F$ is of general type, we have $p_{g}(F) \geq q(F)$. By Proposition 1 , we have that either $p_{g}(F)=1$ or $p_{g}(F)=g(C)=2$. The latter case does not occur since $q(X) \leq g(C)+q(F) \leq 2+p_{g}(F)=4$, contradicting the assumption. Now if $\kappa(\operatorname{alb}(X))=2$, then the image alb $(F)$ of $F$ under the Albanese map of $X$ is a curve of genus $\geq 2$. So $p_{g}(F) \geq q(F) \geq g\left(\operatorname{alb}_{\mathrm{X}}(F)\right) \geq 2$. This is a contradiction.

Example 1. 1) Let $S$ be a smooth surface of general type with $q(S)=2$ such that i) alb: $S \rightarrow$ $\operatorname{Alb}(S)$ is surjective and ii) $\Phi_{S}$ is composed with a pencil (cf. [X2, p. 600] for such examples). Let $X=S \times C$, where $C$ is a smooth curve of genus $\geq 2$. Then $X$ is of Albanese general type and $\operatorname{dim}\left(\operatorname{Im} \Phi_{X}\right)=2$.

2) Let $X=S \times C$, where $S$ is a smooth surface of general type with $p_{g}(S)=q(S)=1$ and $C$ is a smooth curve of genus $\geq 2$. Then $\mathrm{a}(X)=2$ and $\Phi_{X}$ is composed with a pencil.

Remark. 1) The condition $q(X) \geq 6$ in Theorem 1 is technically needed in the proof of Case 2 when $g(C)=1$. When $g(C)=0$, a similar argument of Case 2 works only assuming $q(X) \geq 4$. Thus from our proof we see that Theorem 1 holds if either i) $q(X) \geq 4$ and $g(C)=0$ or $g(C) \geq 3$, or ii) $q(X) \geq 6$ and $g(C)=1$, or iii) $q(X) \geq 5$ and $g(C)=2$.

We conjecture that Theorem 1 is true under the assumption $q(X) \geq 4$.

Examples of 3-fold $X$ of general type whose canonical map is composed with a pencil, with $q(X)=3$ and $a(X)=3$ are easily constructed. Let $(A, \Theta)$ be a principal polarized Abelian $n$-fold $(n \geq 3)$, and $D \in|2 \Theta|$ a smooth divisor. Let $X \rightarrow A$ be the double cover determined by the double cover data $(D, \Theta)$. Then $p_{g}(X)=2$ and $\operatorname{alb}(X) \simeq A$.

2) The condition that $X$ is of general type in Theorem 1 is indispensable. For example, let $X=A \times C$, where $A$ is an abelian variety and $C$ is a smooth curve of genus $\geq 2$. Then $X$ is of Albanese general type and the map induced by $\left|K_{X}\right|$ is composed with a pencil.

3 ) Let $n \geq 3$ be an integer, and $X$ be a smooth projective $n$-fold $X$ of general type and of maximal Albanese dimension. Then it is easy to see that $p_{g}(X) \geq 1$ and by a theorem of Ueno [Ue], one has $p_{g}(X) \geq 2$ if $q(X)>n$.

Question. Is there such an $n$-fold $X$ with $p_{g}(X)=1$ ?

Clearly, a statement similar to Theorem 1 for $(n+1)$-folds of general type is false if the above question has a positive answer.

\section{Deformations of THE CANONICAL MAP}

Let $f: X \rightarrow Y$ be a morphism of smooth projective manifolds with connected fibers. It is known that the morphism $f: X \rightarrow Y$ (of the whole triple) has a semiuniversal deformation. 
Its base space is denoted by $\operatorname{Def}(f: X \rightarrow Y)$. There is a natural map

$$
\mathcal{P}: \operatorname{Def}(f: X \rightarrow Y) \rightarrow \operatorname{Def}(X),
$$

where $\operatorname{Def}(X)$ is the base space of the semiuniversal deformation of $X$.

Fact 1. If $h^{0}\left(R^{1} f_{*} \mathcal{O}_{X} \otimes \mathcal{T}_{Y}\right)=0$, where $\mathcal{T}_{Y}$ is the dual of $\Omega_{Y}$, then $\mathcal{P}$ is surjective.

We refer the reader to [Ca, Il, Fl, Ho, Pa, Ra ] for the basic results on deformations of morphisms and the proof of Fact 1.

Proposition 2. Let $f: X \rightarrow C$ be a fibered 3-fold whose general fiber $F$ is a surface with $p_{g}(F)=1$. Then $\Phi_{X}$ is composed with a pencil if $p_{g}(X) \geq 2$.

Proof. Since $p_{g}(F)=1$ by assumption, $\mathcal{L}:=f_{*} \omega_{X}$ is an invertible sheaf. We have an exact sequence of sheaves

$$
0 \rightarrow f^{*} \mathcal{L} \rightarrow \omega_{X}
$$

with $H^{0}\left(f^{*} \mathcal{L}\right)=H^{0}\left(\omega_{X}\right)$. This implies that $\Phi_{X}$ factors through $f$.

We need the following result. For a proof, see [Be2] or [Si].

Fact 2. (Siu-Beauville) Let $g \geq 2$ be an integer, and $\pi_{1}(g)$ be the fundamental group of a Riemann surface of genus $g$. Let $X$ be a compact Kaehler manifold of dimension $\geq 2$. Then $X$ has a pencil of genus $\geq g$ if and only if there exists a surjective homomorphism from $\pi_{1}(X)$ to $\pi_{1}(g)$.

Theorem 2. Let $X$ be a smooth projective 3-fold of general type whose canonical map is composed with a pencil. Let $f: X \rightarrow C$ be the fiber space associated with $\Phi_{X}$. If $g(C) \geq 2$, then the canonical map of any (global) smooth deformation of $X$ is composed with a pencil.

Proof. Since the geometric genus is a global deformation invariant, the theorem is trivially true when $p_{g}(X)=2$. So we can assume that $p_{g}(X) \geq 3$. By Proposition 1 , we have that $p_{g}(F)=1$, where $F$ is a general fiber of $f$.

Let $X^{\prime}$ be any (global) smooth deformation of $X$, that is, there is a relatively projective smooth family $\pi: \mathcal{X} \rightarrow B$ such that $\mathcal{X}_{b} \simeq X, \mathcal{X}_{b^{\prime}} \simeq X^{\prime}\left(b, b^{\prime} \in B\right)$ and $B$ is connected. Let $t \in B$ be any given point. By Fact 2, there is a surjective morphism $f_{t}: \mathcal{X}_{t} \rightarrow C_{t}$ to a curve $C_{t}$ of genus $\geq g(C)$. Note that $q(X) \leq g(C)+q(F) \leq g(C)+1\left(q(F) \leq p_{g}(F)\right.$ since $F$ is of general type), we have that $g\left(C_{t}\right)=g(C)$ again by Fact 2 . Since

$$
h^{0}\left(R^{1} f_{t *} \mathcal{O}_{\mathcal{X}_{t}} \otimes \mathcal{T}_{C_{t}}\right)=h^{1}\left(R^{1} f_{t *} \omega_{\mathcal{X}_{t}} \otimes \omega_{C_{t}}\right)=0
$$

by [Ko, Theorem 2.1], $\operatorname{Def}\left(f_{t}: \mathcal{X}_{t} \rightarrow C_{t}\right) \rightarrow \operatorname{Def}\left(\mathcal{X}_{t}\right)$ is surjective by Fact 1 , there is a sufficiently small neighborhood $U_{t}$ of $t \in B$, such that for any $t^{\prime} \in U_{t}, \mathcal{X}_{t^{\prime}}$ admits a holomorphic map $\tilde{f}_{t^{\prime}}: \mathcal{X}_{t^{\prime}} \rightarrow \tilde{C}_{t^{\prime}}$ which is a smooth deformation of $f_{t}: \mathcal{X}_{t} \rightarrow C_{t}$. On the other hand, $\mathcal{X}_{t^{\prime}}$ admits at 
most one holomorphic map to a curve of genus $g(C)$ up to isomorphism since $q\left(\mathcal{X}_{t^{\prime}}\right) \leq g(C)+1$. So $\tilde{f}_{t^{\prime}}$ and $f_{t^{\prime}}$ are the same fibrations up to isomorphism. This implies that the geometric genus of the general fiber of $f_{t^{\prime}}: \mathcal{X}_{t^{\prime}} \rightarrow C_{t^{\prime}}\left(t^{\prime} \in U_{t}\right)$ is constant on $U_{t}$. Consequently, since $B$ is connected, we have that $p_{g}\left(F^{\prime}\right)=p_{g}(F)=1$, where $F^{\prime}$ is a general fiber of $f_{b^{\prime}}: \mathcal{X}_{b^{\prime}} \rightarrow C_{b^{\prime}}$. By Proposition 2, we get the result.

Remark. Let $X$ be a smooth complex projective manifold of general type with $p_{g}(X) \geq 3$. Assume that $\Phi_{X}$ is composed with a pencil. In general, the canonical map of the (global) deformation of $X$ is not necessarily composed with a pencil even when $\operatorname{dim} X=2$.

Question. Is there a maximal family (i.e., moduli space component) of smooth projective surfaces of general type with $p_{g} \gg 0$ whose canonical maps are composed with a pencil?

\section{REFERENCES}

[Be1] Beauville, A., L'application canonique pour les surfaces de type général, Invent. Math. 55 (1979), 121140.

[Be2] Beauville, A., Appendix to [Ca].

[BPV] Barth, W., Peters, C., Van de Ven, A., Compact complex surfaces, Ergeb. Math. Grenzgeb., 1984.

[Ca] Catanese, F., Moduli and classification of irregular Kaehler manifolds (and algebraic varieties) with Albanese general type fibrations, Invent. Math. 104 (1991), 263-289.

[CC] Chen, M., Chen, Z. J., Irregularity of canonical pencils for a threefold of general type, Math. Proc. Camb. Phil. Soc. 125 (1999), 83-87.

[Fl] Flenner, H., Über deformationen holomorpher abbildungen, Osnabrücker Schriften zur mathematik I-VII and 1-142, 1979.

[Fu] Fujita, T., On Kaehler fibre spaces over curves, J. Math. Soc. Japan 30 (1978), 779-794.

[Ha] Hartshorne, R., Algebraic Geometry, GTM 52, Springer-Verlag, 1977.

[Il] Illusie, L., Complexe cotangent et deformations I, II, LNM 239, LNM 283 Springer-Verlag, (1971).

[Ho] Horikawa, E., On deformation of holomorphic maps, I, II, J. Math. Soc. Japan vol 25 (1973), 372-397; vol 26(1974) 647-667; III, Math. Ann. 222, 275-282 (1976).

[Ko] Kollár, J., Higher direct images of dualizing sheaves I, Ann. Math. 123 (1986), 11-42.

[Pa] Palamodov, V.P., Deformations of complex spaces, Usp. Mat. Nauk. 31 (1976), 129-194.

[Ra] Ran, Z., Deformations of maps, LNM 439, Springer-Verlag, (1989), 246-253.

[Mo] Mori, S., Classification of higher-dimensional varieties, Proceedings of Symposia in Pure Math. 46 (1987), 269-331.

[Si] Siu, Y. T., Strong rigidity for Kaehler manifolds and the construction of bounded holomorphic functions, in Discrete groups and analysis (Howe, R., eds.), Birkh..auser, 1987, pp. 124-151.

[Ue] Ueno, K., Classification theory of algebraic varieties and compact complex spaces, LNM 439, SpringerVerlag, (1975).

[X1] Xiao, G., L'irregularité des surfaces de type général dont le système canonique est composé d'un pinceau, Comp. Math. 56 (1985), 251-257.

[X2] Xiao, G., Irregularity of surfaces with a linear pencil, Duke Math. J. 55 (1987), 597-602. 